\section{METABOLISM}

\section{Bone hormone boosts exercise}

A hormone released from bones enhances muscle function during exercise, giving old mice the capabilities of young ones.

Gerard Karsenty of Columbia University Medical Center in New York City and his colleagues found that blood concentrations of a hormone called osteocalcin increased during aerobic exercise in mice, monkeys and people. The hormone helped muscles to adapt to exercise by increasing their uptake and use of glucose and other nutrients. Mice that lacked the gene for osteocalcin had diminished exercise capacity.

Blood concentrations of osteocalcin declined as animals aged, and administering the hormone to 15-month-old mice gave them the exercise capacity of 3-month-old animals. Cell Metab. 23, 1078-1092 (2016) ASTRONOMY

\section{Early galaxy has wisps of oxygen}

Astronomers have detected oxygen in a 13-billion-yearold galaxy - the first time that the gas has been found at such an early stage of the Universe.

A team led by Akio Inoue at Osaka Sangyo University in Daito, Japan, used the powerful Atacama Large Millimeter/submillimeter Array (ALMA) in Chile to measure the chemical make-up of the galaxy, which was discovered in 2012.

Oxygen was only one-tenth as abundant as it is in the Sun, and the galaxy seemed to be

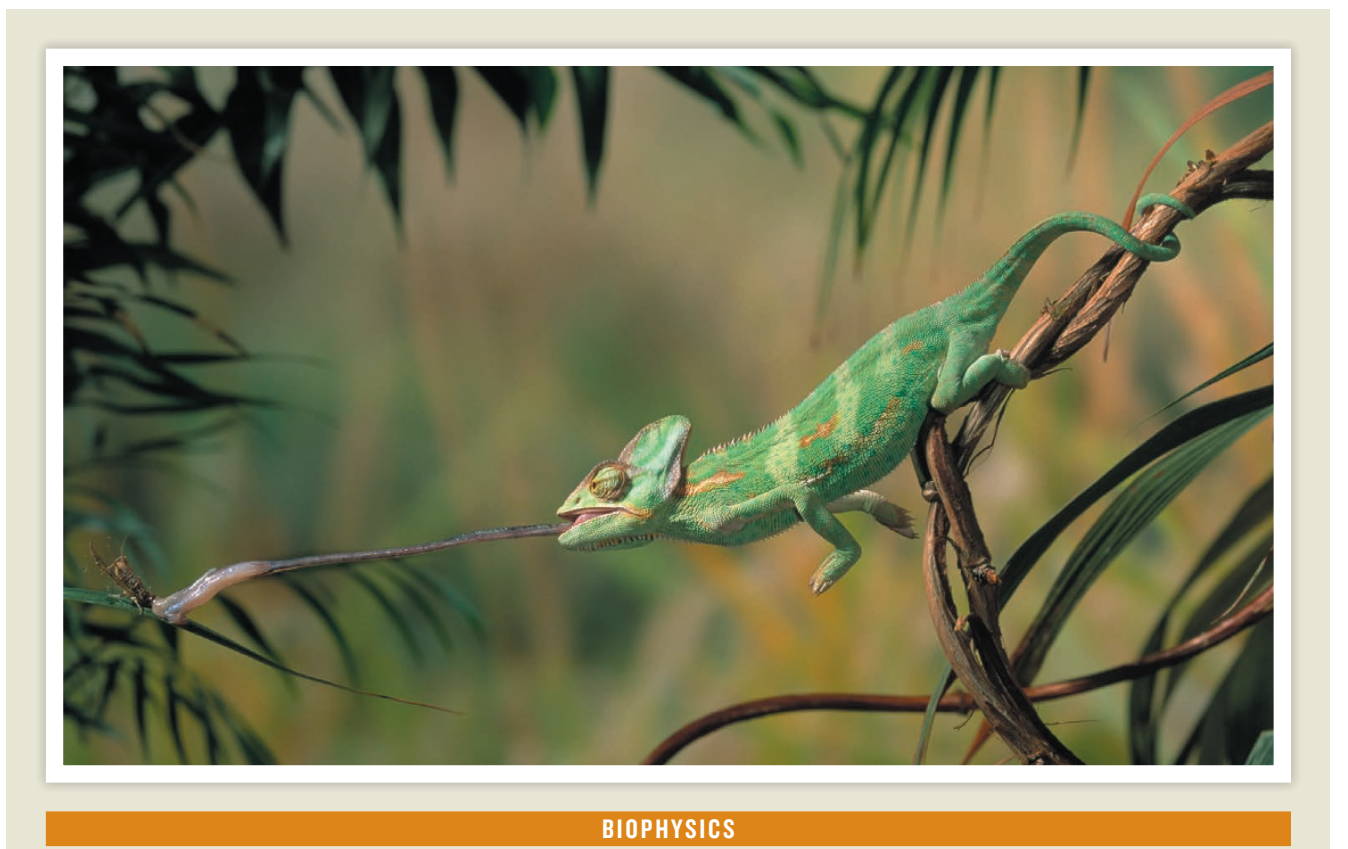

\title{
Chameleons' sticky spit grabs prey
}

Adhesive mucus allows chameleons to snare insects with their long tongues.

Pascal Damman at the University of Mons in Belgium and his colleagues collected mucus from the tongue pads of veiled chameleons (Chamaeleo calyptratus; pictured) and found that it is 400 times more viscous than human saliva. Using a model of chameleon tongue strikes, the team estimated that the mucus allows the animal to capture insects that are up to $60 \%$ of its body size - larger than its natural prey.

The size of prey a chameleon can nab is therefore not limited by the stickiness of its tongue, the authors say.

Nature Phys. http://dx.doi.org/10.1038/nphys3795 (2016) low in neutral gas and dust.

Such characteristics may have allowed ultraviolet light from the stars of this and other similar galaxies to escape and ionize the hydrogen atoms in the early Universe, eventually generating the levels of ions seen today.

Science http://doi.org/bj5z (2016)

\section{NEUROSCIENCE}

\section{Smart birds have big brains}

Birds that sing or use tools have about as many neurons in their brains as monkeys do. Pavel Němec at Charles University in Prague and his colleagues measured the brain size of birds from 28 species, and counted the number of cells in the organs. They found that intelligent birds such as parrots and songbirds have larger brains relative to their body size, with much higher neuron density, than do less-intelligent birds such as chickens. Moreover, a higher proportion of the neurons were located in the forebrain, which controls higher cognitive function.

Such high neuronal densities could be contributing to the intelligence of the birds, the authors suggest.

Proc. Natl Acad. Sci. USA http://doi.org/bjzx (2016)

\section{Microbe makes mice social}

Female mice that eat a high-fat diet produce litters with social deficits that are linked to changes in the offspring's gut bacteria.

Mauro Costa-Mattioli at Baylor College of Medicine in Houston, Texas, and his colleagues compared offspring from mothers that ate a high-fat diet with those from mothers on a normal diet. The high-fatdiet offspring spent less time interacting with other mice, and had reduced bacterial diversity in their 